\title{
LA COMERCIALIZACIÓN DEL TURISMO INDUSTRIAL
}

\author{
Iria Caamaño Franco
}

Universidade da Coruña

\begin{abstract}
Resumen: En este artículo se plantea la creación de un producto turístico comerciable a partir de un recurso industrial, ya bien sean instalaciones abandonadas o una empresa en activo. Como introducción se parte de la juventud de esta tipología turística y se expone una breve descripción del turismo industrial así como de los recursos relacionados con el mismo.

A continuación, se ofrecen unas pautas a seguir para convertir ese recurso en un producto turístico atractivo y que se pueda comercializar, exponiendo simultáneamente ejemplos de éxito. Además se establecen las ventajas y desventajas que este producto ofrece tanto a las empresas (activas o inactivas) como a los propios destinos en su desarrollo.

Finalmente, a partir del contexto en el que se encuentra la comercialización, dentro del marco de las nuevas tecnologías, se establecen las posibles vías de comercialización de productos turísticos relacionados con el turismo industrial.
\end{abstract}

Palabras clave: Turismo industrial, producto turístico, comercialización, destinos turísticos, diversificación de la oferta.

Resumo: A comercialización do turismo industrial

Neste artigo exponse a creación dun produto turístico comerciable a partir dun recurso industrial, ben sexa unha instalación abandonada ou ben unha empresa en activo.

Como introdución pártese da novidade desta tipoloxía turística e faise unha breve descrición do turismo industrial, así como dos recursos con el relacionados. A seguir, ofrécense unhas pautas para converter un deses recursos nun produto turístico atractivo e que se poida comercializar, ao tempo que se poñen exemplos de éxito. Ademais establécense as vantaxes e desvantaxes que este produto ofrece tanto ás empresas (activas ou inactivas) como aos propios destinos no seu desenvolvemento.

Finalmente, a partir do contexto en que se atopa a comercialización, dentro do marco das novas tecnoloxías, establécense as posibles vías de comercialización de produtos turísticos relacionados co turismo industrial.

Palabras clave: Turismo industrial, produto turístico, comercialización, destinos turísticos, diversificación da oferta.

Abstract: The commercialisation of industrial tourism 
This paper considers the creation of a commercialisable tourist product based on an industrial resource which may either be disused facilities or an active industry.

It begins by considering the relatively recent nature of this phenomenon, including a brief description of industrial tourism and its related resources.

The text then goes on to provide a series of guidelines aimed at turning this resource into an attractive tourism product suitable for commercialisation, providing several examples of successful initiatives. It also considers the advantages and disadvantages these products represent for companies (active or inactive) and the development of the locations in which they are situated.

Finally, and within the context of commercialisation and the framework of new technologies, it considers the various forms of commercialisation for tourism products related to industrial tourism.

Key words: industrial tourism, tourist product, commercialisation, tourist destinations, supply diversification

\section{APROXIMACIÓN AL CONCEPTO}

En la actualidad las tendencias turísticas están sufriendo una evolución, a veces, más rápido de lo que podemos llegar a percibir. Muchos son los autores, entre ellos García y Rivas (2007), que hacen referencia a un cambio en los gustos, tanto estéticos como turísticos de la sociedad, distinguiendo de este modo entre el turismo tradicional de masas y el turismo alternativo.

Dentro del turismo tradicional de masas encontramos el ya consolidado turismo de sol y playa, considerado como menos exigente y menos especializado. El turismo alternativo por su parte inherentemente lleva un sello de calidad que es exigido por una amplia parte de la demanda y que busca nuevas modalidades como pueden ser el turismo rural, el turismo náutico, el turismo cultural... (Castillo et al., 2011),

A su vez, el turismo cultural no sólo implica la visita a monumentos o lugares de interés sino que también hace referencia al consumo del modo de vida (Fernández y Guzmán, 2005) convirtiéndose en un concepto muy amplio en el que podemos incluir el turismo gastronómico, el turismo religioso... o el turismo industrial.

Tal y como podemos observar, existe una gran problemática a la hora de establecer unos parámetros para diferenciar las tipologías turísticas unas de otras. Asimismo, no se descarta una posible complementariedad entre los diferentes tipos de turismo, como por ejemplo entre el tradicional turismo de sol y playa y el turismo cultural en todas sus variantes (García y Alburquerque, 2003).

\section{TURISMO INDUSTRIAL}

El patrimonio industrial es el más joven de todos los patrimonios puesto que abarca un conjunto de estructuras, piezas y máquinas que han sido utilizadas en muchos casos hasta fechas recientes (Pardo, 2004). 
En España, el Plan Nacional de Patrimonio Industrial desarrollado por el Ministerio de Cultura define el patrimonio industrial como "el conjunto de elementos de explotación industrial generado por las actividades económicas de cada sociedad que responde a un determinado proceso de producción y a un sistema tecnológico concreto caracterizado por la mecanización dentro de un sistema socioeconómico". (www.incuna.es)

Dentro del patrimonio industrial podemos encontrar zonas de producción, de vivienda, etc., así como objetos inmuebles (como las maquinarias, herramientas, atractivos, etc.,) y modos de vida de los trabajadores o el know how de los procesos productivos (Fernández y Guzmán, 2005).

El turismo relacionado con la visita a dicho patrimonio es también reciente. El término turismo industrial surge en los años 40 en Francia, años en los que ya se comienzan a desarrollar visitas a bodegas y a fábricas de chocolate en dicho país. Sin embargo, hace más de cien años que tanto las organizaciones privadas como públicas realizan visitas a industrias (Otgaar et al., 2010).

La temprana edad de dicho término provoca que exista un claro problema terminológico alrededor del concepto "turismo industrial", siendo multitud las actividades a las que puede hacer referencia dicho término y, por tanto, múltiples las disciplinas que estudian y plantean sus propias definiciones. Además, a la hora de plantear una definición tenemos que presentar ciertos matices dependiendo de los países en los que utilicemos el término, puesto que el significado puede llegar a cambiar sustancialmente. En países como Francia, Reino Unido, Alemania o Suecia el turismo industrial se encuentra ya consolidado, presentando una definición con muchos más matices.

Según Edwards y Llurdés, citados por García y Rivas (2007), el turismo industrial se puede definir como "el desarrollo de actividades turísticas e industrias en lugares edificados por el hombre, edificios y paisajes que se originan en procesos industriales de tiempo pasados".

Una de las definiciones más actuales la ofrecen Otgaar et al. (2010) quienes simplifican en una definición más sencilla el término turismo industrial, sirviendo de este modo como resumen de todas las anteriores definiciones. Para dichos autores el turismo industrial se resume en visitas a empresas que aún siguen en funcionamiento y al patrimonio industrial, acogiendo así ambos conceptos en un solo término.

Sin embargo, en muchas ocasiones relacionan el turismo industrial exclusivamente con las visitas al propio patrimonio (fábricas en desuso, bien sea en su estado abandonado o reconstruidas), no incluyendo en dicho término las visitas a empresas vivas. Del mismo modo, en España, Valls et al. (2004) se refiere al turismo industrial urbano como aquel que está basado en la arqueología industrial característica de la revolución industrial de finales del siglo XVIII y XIX.

Otro de los aspectos negativos del término a los que se puede hacer referencia es que él mismo se contradice, haciendo referencia a su vez a dos términos totalmente contrarios. Por un lado, al ocio y al descanso (turismo) y por otro lado, al trabajo (industrial). 
De este modo, estamos ante un término confuso, llegando a influir negativamente tanto en el propio sector como en la decisión del turista.

\section{PRODUCTO TURÍSTICO}

Sin darnos cuenta en muchos de los viajes vacacionales tradicionales se realizan visitas relacionadas con el turismo industrial, visitando por ejemplo centros de interpretación o museos que se encuentran en antiguas fábricas de salazón, industrias textiles, minas...

En la mayoría de las ocasiones nos hemos encontrado con el recurso de manera fortuita, ningún tipo de promoción y sin una correcta comercialización. Por ello, a continuación se presentan las razones por las cuales es necesaria la creación de un producto turístico industrial.

Existen todavía muchos recursos turísticos que no han sido convertidos en productos turísticos y que, por tanto, están perdiendo su real potencial. Una vez que tenemos identificado el recurso, el siguiente paso es ponerlo en valor para poder crear un producto que finalmente sea comercializado.

Teniendo en cuenta que un elemento cultural en sí mismo no es un recurso turístico, es necesario seguir ciertos pasos para convertirlo finalmente en un producto que, a su vez, buscamos que sea atractivo.

El primer paso puede ser catalogarlo como patrimonio consiguiendo de este modo conservarlo y posicionarlo en la mente de los consumidores, proporcionándole cierta categoría al recurso.

Debido a la inmensa cantidad de restos patrimoniales relacionados con la industria, a la hora de seleccionar nuestro recurso muchos son los autores que optan por aplicar políticas de conservación selectivas. Para ello es aconsejable que un equipo de expertos realice un análisis pluridisciplinar sobre el potencial turístico de ese patrimonio.

Una vez que obtengamos los resultados del análisis, valoraremos. Si el potencial turístico es alto, es importante que dichos elementos sean catalogados con alguna protección como, por ejemplo, bienes de interés cultural. De dicho modo estará protegida su conservación y posteriormente se podrá, y se deberá, darle uso.

El siguiente paso, en muchos casos olvidado, será transformar el recurso en un producto turístico. En este caso, el recurso debería estar gestionado por agentes (ya bien sean públicos, privados o mixtos), puesto que sería la forma más adecuada de posteriormente lanzarlo al mercado turístico. De todos modos, hay que ser conscientes de la dificultad de la creación de un producto turístico, ya que requiere un amplio conocimiento del recurso y del mercado por parte de dichos agentes.

Lobo (1998) señala como características más relevantes del producto su intangibilidad, su elaboración y adquisición en un lugar distinto a aquel en el que se disfruta, su imposibilidad de almacenamiento, su pago generalmente de forma previa y 
su venta en puntos especializados.

Buscando un mayor beneficio del producto turístico tenemos que tener presente una de las características con las que todo destino, y agente del sector turístico, debe de buscar combatir: la estacionalidad.

La estacionalidad es una de las características inherentes al desarrollo del turismo y, aunque hablemos de un producto alternativo como es el turismo industrial, tenemos que tenerlo presente a la hora de comercializarlo. De todos modos, somos conscientes de la problemática que resulta combatirla.

Por tanto, es necesario tener en cuenta que dependiendo del mes del año nuestro público será diferente $\mathrm{y}$, por tanto, nuestro producto también debe de ser diferenciado.

En temporada baja las visitas suelen ser realizadas, mayoritariamente, por gente que busca formarse en diferentes aspectos: sobre la tecnología utilizada, el conocimiento de la empresa, el propio proceso... Por un lado, podemos tener las visitas realizadas por grupos de escolares, universitarios... en muchas ocasiones nuestro público las estará realizando por obligación por lo que es muy importante prepararles una visita adaptada para captar su atención y posibles futuros clientes. Por otro lado, incluso también los propios científicos o trabajadores de la propia compañía pueden realizar visitas a las instalaciones.

En temporada alta el tipo de turismo que se puede ver atraído es individual o grupos organizados que buscan en su tiempo de ocio ver, o incluso probar, los productos, conocer el proceso... En definitiva, a la mayoría nos gusta conocer cómo y dónde se fabrican todas aquellas cosas que diariamente nos rodean y por ese motivo somos muchos los que invertimos parte de nuestro tiempo libre en descubrirlas.

Podemos solucionar dichos problemas de estacionalidad gracias a la gran variedad de productos que ofrece el turismo industrial. Todo ello nos permitirá tener a un público más amplio, aunque también debemos ofrecer unos productos más específicos.

Además de tener en cuenta dichas características inherentes a todo producto turístico, a continuación planteamos algunas de las principales características que consideramos son imprescindibles tenerlas presente a la hora de elaborar un producto, con el objetivo de que nuestro producto tenga éxito en el mercado: 
Cuadro 1: Características del producto

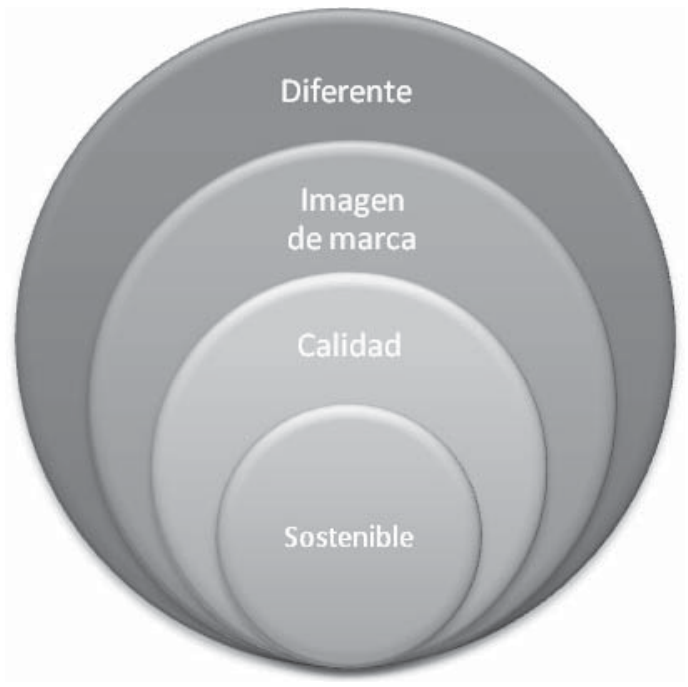

Fuente: Elaboración propia

Diferente. En primer lugar, necesitamos que nuestro producto sea diferente. Lo ideal es crear un producto turístico con atractivos que lo hagan único pero de todos modos es importante que lo que vayamos a enseñar, explicar o exponer sea un proceso de fabricación llamativo.

Uno de los ejemplos más llamativos es la visita a una central nuclear y varios ejemplos atestiguan el éxito turístico de dichas instalaciones. Por un lado destacar la central de Bataan, situada a dos horas de Manila (Filipinas) la cual fue construida por Ferdinand Marcos hace más de 30 años y ahora se ha convertido en uno de los recursos turísticos más importantes de la zona. Por otro lado, el mismo éxito ha ocurrido en Chernóbil quien se ha convertido en uno de los diez destinos más visitados de Ukrania después del grave accidente ocurrido en 1986.

Otra forma de conseguir llegar a ser un producto atractivo, en el caso de que se decida rehabilitar una fábrica, es conseguir que la rehabilitación sea llevada a cabo por arquitectos reconocidos puesto que dicha acción aportará a nuestro producto un valor añadido y un reclamo más para el turista. Sin embargo, somos conscientes de que dicha estrategia debe contar con una importante inversión económica que no todos se pueden permitir. Un ejemplo de dicho caso es Codorníu, en la provincia de Barcelona la cual se caracteriza por ser un lugar diferente estéticamente y cuya bodega ha sido catalogada como Monumento Histórico Artístico desde 1976 por ser obra de uno de los representantes más importantes de la escuela modernista catalana, el arquitecto Josep Puig i Cadafalch.

Además, no nos podemos olvidar que es muy importante tener siempre actividades y exposiciones itinerantes atractivas. Muchas de estas actividades en la mayo- 
ría de las ocasiones quedan simplemente destinadas a la población local y son muy pocos los turistas que realmente se interesan o realizan finalmente estas actividades, por lo que nuestro objetivo será implicar a ambos públicos de igual modo.

Imagen de marca. Otro de los elementos necesarios que debe de tener nuestro producto, y que del mismo modo lo posicionará rápidamente, es la importancia de la historia de la fábrica o, si está en activo la empresa, su propia marca. En definitiva, la imagen de la marca conseguirá que el producto sea fácil de reconocer y de quedar posicionado, convirtiéndose en uno de los productos estrella del destino. Por ejemplo, una de las empresas más visitadas en Estados Unidos es la fábrica de Coca Cola en Atlanta. En el caso de Irlanda, la visita a la capital no se concibe sin pasar por la fábrica de Guinnes Storehouse.

Si en nuestro caso aún no tenemos una imagen de marca potente deberemos de hacer campañas de promoción para conseguirlo.

Calidad. Necesitamos of recer calidad para convertirnos en un producto más competitivo. En esta dirección se ha estado trabajando durante los últimos años, tanto a nivel particular por parte de las empresas como a nivel general en el sector.

Por un lado, la organización internacional de normalización (International Organization for Standardization, conocida más comúnmente como ISO) aprobó una norma de calidad para el turismo industrial que es la TC 228/WG 9.

Por otro lado, desde el ICTE se está luchando para conseguir a nivel nacional una norma española de turismo industrial con el fin de normalizar las experiencias turísticas vinculadas a actividades industriales, científicas y técnicas actuales o relacionadas con otros momentos y épocas históricas.

Sostenible. Ofrecer un producto sostenible es un claro elemento diferenciador. Sin embargo, en la mayoría de las ocasiones no se sabe vender adecuadamente este elemento. En este caso, un producto de turismo industrial se relacionará rápidamente con la contaminación que la industria de por sí provoca, por lo que será aún más necesario una adecuada transmisión de este valor.

Es importante recalcar el impulso hacia la sostenibilidad que supone la conservación de patrimonio industrial pero también es necesario una buena gestión y promoción para el desarrollo de un turismo sostenible.

En definitiva, los destinos que decidan reconstruir zonas industriales o incluso la propia fábrica en un nuevo centro de interpretación, museo etc... deberán de plantearse desde el principio hacer atractivo un producto, diferenciarse, ofrecer calidad o por su imagen de marca.

A continuación se expone la clasificación de los diferentes productos que podemos encontrar dentro del turismo industrial: 
Cuadro 2: Productos de turismo industrial

Reliquias industriales en el

ámbito de la producción y

de los procesos de trabajo.

- Minas

- Embarcaderos

- Molinos...

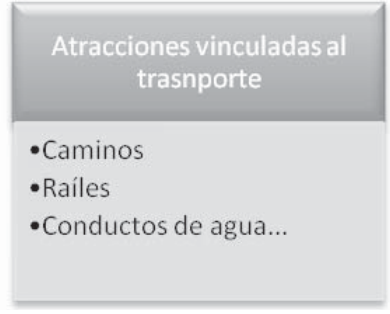

Atracciones socioculturales vinculadas al pasado de una región particular

- Casas y lugares de recreación de los trabajadores

Fuente: Elaboración propia a partir de Hospers (2002)

Dicho patrimonio ha perdido su uso original debido a la paralización del sector industrial, por lo que urge darle nuevos usos. Para ello se presentan normalmente restaurados y convertidos en lugares para conciertos, centros socio culturales, museos de arte contemporáneo, exposiciones sobre la historia de la fábrica y de la revolución industrial... o en su estado original para mostrar la estética de la desindustrialización.

La reutilización y puesta en valor de dichos recursos podrá ayudar a mejorar la imagen degradada de estas zonas y llegar a convertirse en un lugar muy atractivo para los turistas, a pesar de que en muchas ocasiones dichas ciudades se presentan como ejemplo de feísmo. Sin embargo, es importante relativizar los conceptos de belleza y estética en estas ciudades, tal y como señalan Castillo et al. (2011).

\section{VENTAJAS Y DESVENTAJAS DE COMERCIALIZAR PRODUCTO DE TURISMO INDUSTRIAL}

En cuanto a las ventajas y desventajas de los productos de turismo industrial es necesario establecer una diferenciación entre las empresas que siguen en activo y las empresas que no (de las cuales sólo conservamos el edificio o en algunos casos cierta maquinaria).

A continuación, presentamos algunas de las ventajas y desventajas que ofrece el turismo industrial a las empresas que siguen en activo.

La comercialización de un producto industrial puede ofrecer muchas ventajas a una empresa, aunque también es necesario establecer en una balanza junto con las posibles desventajas. Según Frew (2000), citado por Otgaar et al. (2010), podemos resumir las ventajas fundamentalmente en tres puntos:

1. Mejorar su imagen. Dar a conocer las instalaciones de una empresa así como el modo de trabajo. La oportunidad que las visitas les ofrecen, tanto para mejorar la imagen corporativa como para dar a conocer su producto, son incentivos que los gerentes de las empresas valoran positivamente. Además, incluso pueden aprovechar las visitas para dar a conocer productos nuevos 
que aún no son conocidos en el mercado y de este modo llegar a incitar su compra posteriormente, provocando un mayor número de ventas $\mathrm{y}$, por tanto, un mayor número de ingresos.

2. Mejorar la moral de trabajo. Recibir visitantes a la empresa, y que éstos queden satisfechos, implicará un mayor reconocimiento social de la misma. En esta línea, los trabajadores se sentirán orgullosos de la empresa donde trabajan provocando una mejora del reconocimiento social de la misma en la sociedad.

3. Obtener un ingreso extra. El ingreso puede venir por dos vías ya bien sea de manera directa o indirecta. Por un lado tenemos las empresas las cuales cobran por la realización de la visita a sus instalaciones y por otro, tenemos aquellas empresas (mayoritariamente agroalimentarias o artesanales) las cuales ofrecen una venta directa de su producto a los visitantes. Mayoritariamente las empresas más pequeñas no suelen cobrar entrada por realizar su visita sino que buscan aumentar su número de ventas directas al cliente, así como las ventajas citadas anteriormente.

Aunque las ventajas presentadas anteriormente podrían llevar a decidir desarrollar actividades turísticas, muchas empresas debido a su propia actividad cuentan con limitaciones e inconvenientes a la hora de enseñar sus instalaciones. Las principales desventajas son las siguientes:

1. Limitaciones de seguridad. Muchas empresas, debido a la actividad que desarrollan en sus instalaciones tienen prohibida la entrada de gente ajena a las mismas. Ejemplo de ello son aquellas empresas que manipulan alimentos, como por ejemplo las conserveras. Además, algunos departamentos deben estar fuera del alcance de los visitantes simplemente porque la presencia de los mismos perturba el proceso de producción (por ejemplo, en un taller de pintura o en un laboratorio) o porque es peligroso para los propios visitantes estar allí. Sin embargo, dicha desventaja se puede eliminar con ideas innovadoras como la instalación de un túnel que permita la visita y al mismo tiempo evite el contacto con la fábrica.

2. Posible espionaje por parte de la competencia. En un mercado tan competitivo como en el que nos encontramos actualmente, las empresas tienen miedo a que trabajadores de la competencia estén interesados en la visita a sus instalaciones con el fin de vigilar o copiar el producto o el proceso de producción.

3. Incomodidad de los trabajadores. Aunque planteábamos las visitas como una ventaja a la hora de mejorar la moral de los trabajadores también es cierto que se pueden llegar a sentir incómodos al recibir visitas, sentirse observados, distraerse... y, por tanto, no realizar adecuadamente su trabajo.

Tal y como señalan Otgaar et al. (2010), e incluso la propia Unión Europea, el turismo industrial no sólo beneficia a las empresas sino también a la propia ciudad dónde se desarrolle la actividad. En efecto, la realización de dichas visitas puede llegar a convertirse en una estrategia de negocio para la propia empresa e incluso un 
elemento de desarrollo para el destino.

El día 27 de septiembre es el día europeo del turismo y en el año 2011 ha estado dedicado al turismo industrial. Lo que se ha intentado divulgar con las diferentes conferencias presentadas en dicho día ha sido la importancia de la diferenciación de los destinos y el desarrollo sostenible. Además, se ha resaltado la importancia del desarrollo del turismo industrial para la conservación de los testimonios de una parte importante de la historia de Europa, así como la creación de puestos de trabajo.

Es necesario también hacer referencia a algunas de las ventajas que ofrece el turismo industrial para los propios destinos. A continuación se presentan algunas:

1. Conservar la arquitectura industrial. Mayoritariamente la arquitectura industrial que se conserva es del siglo XX. Únicamente se suele conservar la chimenea de la industria en su memoria, las colonias industriales así como algunos instrumentos y maquinaria. Un ejemplo de ello se da en Galicia, concretamente en Serantes (Laxe) donde encontramos los pocos restos de las minas de caolín abiertas en 1923 en este lugar. Actualmente se ha creado un documental "Kaolines de Lage" para recordar la historia de dicha mina.

2. Mejorar la imagen del destino. Una vez que se ponga en valor dicho patrimonio la imagen del destino podrá verse mejorada desde el punto de vista tanto por parte de la propia población local como para los turistas.

3. Diversificar la oferta. Por un lado, a los destinos ya desarrollados turísticamente ofrece diversificar la oferta del destino pudiendo de este modo captar nuevos turistas o incluso mantener los actuales. Por otro lado, a los destinos no desarrollados le permite convertirse en un nuevo destino turístico, buscando al mismo tiempo diferenciarse de otros destinos considerados más tradicionales.

Como desventajas:

1. Poca originalidad. Uno de los principales problemas con los que nos encontramos hoy en día a la hora de crear un producto es la estandarización del mismo. La mayoría de los destinos repiten el mismo producto y no se diferencian de la competencia. En definitiva, al no ser selectivos y no crear un producto diferente estaremos malgastando el dinero en la rehabilitación pero sin convertirlo realmente en un producto turístico rentable, que en primera instancia es lo que se busca a la hora de crear un producto turístico.

2. Poca promoción. Acabada ya la fase de puesta en valor del recurso, en la mayoría de las ocasiones la más costosa, posteriormente no se invierte lo suficiente en la promoción del producto. Aunque si bien es cierto que sí que se puede llegar a invertir en promoción, se realiza de modo descontrolado. En muchas ocasiones se ha invertido y se han hecho campañas sin un estudio previo del cliente al que queremos dirigirnos y por tanto, ante una situación de malgasto de presupuesto.

3. Poca cooperación. La mayoría de los inconvenientes de la puesta en marcha de un producto turístico radican en la poca cooperación entre las diferentes administraciones y las empresas privadas. 
En referencia al patrimonio industrial inactivo, la utilización del mismo para el turismo es una forma de darle un nuevo uso y al mismo tiempo de rentabilizarlo. Dicha responsabilidad puede llevarse a cabo por parte de un ente privado o, como suele ocurrir en la mayoría de las ocasiones, por parte de un ente público.

En busca de impulsar un desarrollo, planificado y sostenible del turismo, la Asociación Euroeume, en el marco del Programa PRODER, promovió la redacción del Plan de Desarrollo Turístico (PDT). En el Plan de Desarrollo Turístico del Eume (1998) se proponía un modelo turístico basado en el turismo rural y el turismo industrial, considerando dicho patrimonio como un elemento que contribuía a la diversificación económica del territorio. El objetivo principal era promulgar el patrimonio tecnológico e industrial en este caso vinculados al eje temático del aprovechamiento de la energía (centrales hidroeléctricas, mina, central Endesa...).

Posteriormente, el Plan de Dinamización del Producto Turístico Ferrol-Ortegal (2006) también ha desarrollado un pequeño folleto con rutas relacionadas con el turismo industrial. En dicho folleto encontramos varias rutas en las que el turista puede conocer diferentes ayuntamientos del destino diversificando la oferta.
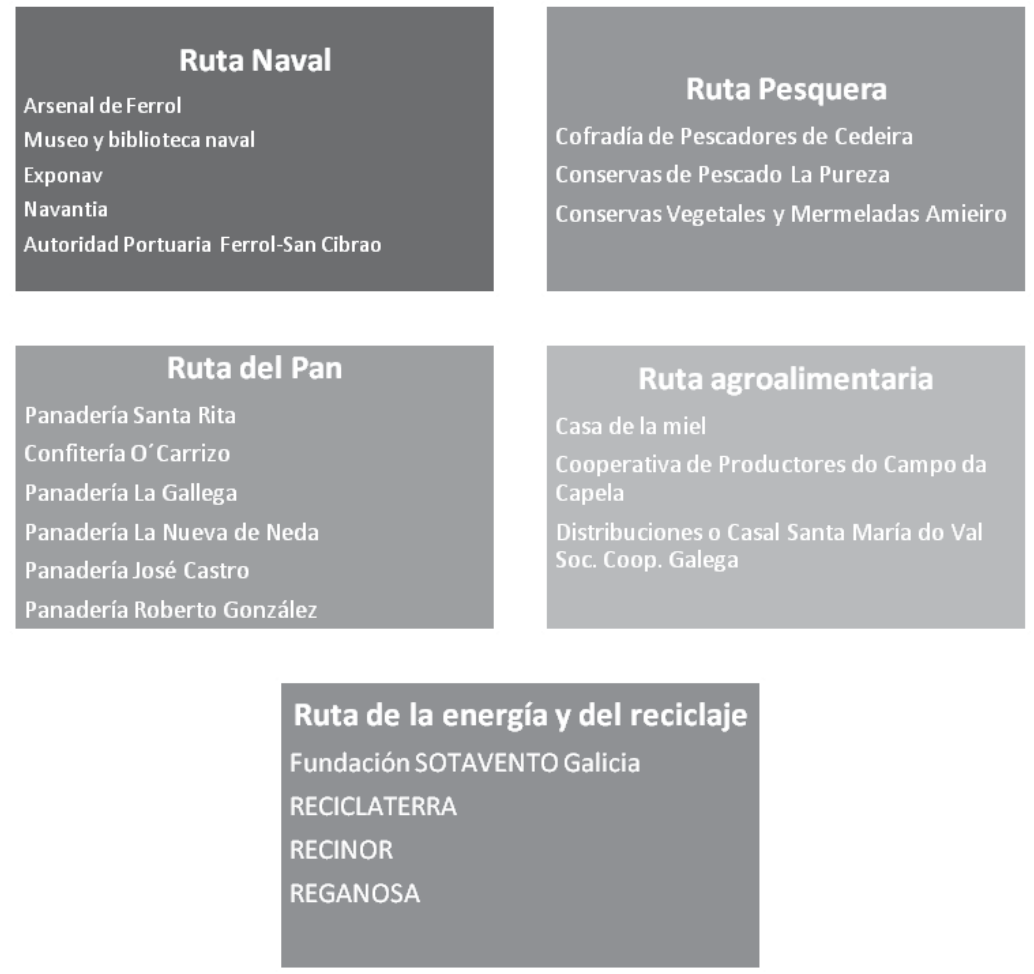

Ruta naval. Gracias a esta ruta el turista puede conocer diferentes partes del puerto de Ferrol, íntegramente ligado a la historia de esta ciudad. De este modo el turista conoce la ciudad pero desde un punto de vista totalmente innovador. 
Ruta pesquera. Galicia se caracteriza sobre todo por su relación con el mar. Por este motivo, en el destino Ferrol-Ortegal se permite conocer el puerto, la lonja y una fábrica de elaboración de salazones y conservas de pescados de forma artesanal.

Ruta agroalimentaria. En esta ruta se permite conocer varios productos gastronómicos del destino a través de su producción. Una de las iniciativas más interesantes es la casa de la miel.

Ruta del pan. Se ha desarrollado una ruta con posibilidad de participar en un taller de elaboración de pan en un molino rehabilitado. Dichas visitas deben de pedirse con cita previa y permiten al turista conocer el proceso tradicional de elaboración del pan, amasándolo y llevándolo como suvenir. Varias son las panaderías de la zona que quisieron formar parte de esta ruta.

Ruta de la energía y del reciclaje. Esta propuesta para conocer la energía, el reciclaje y su relación con el medio ambiente es una ruta atractiva no sólo para los turistas que llegan al destino sino también para la propia población local.

En general, esta iniciativa ha supuesto un avance en esta tipología turística, convirtiéndose en una iniciativa innovadora en Galicia y extrapolable a otras comarcas.

Como ya se ha comentado anteriormente en el presente artículo, las ciudades industriales siempre han desarrollado una imagen negativa para los turistas e incluso para la propia población local. El desarrollo de un turismo enfocado a la puesta en valor de dichas industrias y de dicho patrimonio (tanto tangible como intangible) conseguirá reinvertir esa imagen en positiva, ofreciendo muchos más beneficios y eliminando la consideración de las ciudades industriales como ejemplo de feísmo.

Un ejemplo muy llamativo es la ciudad de Paterson, ubicado en el río Passaic en Nueva Jersey (EEUU). Paterson fue una de las más poderosas ciudades industriales de los Estados Unidos por lo que conserva una rica historia como primera ciudad planificada de la nación industrial, además de contener algunas de las fábricas más antiguas del país y varias empresas textiles.

Hoy en día Paterson se ha convertido en una ciudad con una imagen negativa para el resto de la población. Esta ciudad es un claro ejemplo de que a pesar de conservar muchas de las instalaciones y tener un increíble potencial turístico no ha sabido aprovecharlo, exceptuando la creación de un museo.

\section{LA DEMANDA}

Una vez que decidimos crear el producto necesitamos establecer unos parámetros. Entre ellos tenemos la necesidad de tener presentes las características presentadas en el Cuadro 1: Características del producto. Sin embargo, también será necesario establecer a qué tipo de turista nos queremos dirigir, diferenciando en primer lugar entre los turistas alternativos generalistas y aquellos que son más especializados (Nácher, 1997). 
El turismo industrial aún sigue siendo una tipología minoritaria a pesar de que cada vez la conoce y la práctica más gente. Uno de los motivos por los que sigue creciendo el número de turistas en busca de turismo industrial es el gran interés por conocer la historia de los siglos XIX y XX, así como los cambios sociales que se produjeron tras la revolución industrial.

Hoppers (2002) distingue dos tipos de turistas que pueden ser atraídos por el turismo industrial. En primer lugar están los veteranos, generaciones que buscan la nostalgia en aquellas visitas a sus antiguos lugares de trabajo (convertidos en museos, centros de interpretación...). Es decir, la gente que ha trabajado alguna vez en una fábrica le gusta visitarla para ver cómo ha evolucionado desde que dejó de trabajar en ella, nuevos avances tecnológicos, la nueva gente que trabaja en ella...

En segundo lugar, las nuevas generaciones que se apasionan por la historia y a las cuales su desconocimiento sobre el funcionamiento de las empresas les provocan una gran curiosidad, tanto sobre el propio proceso de producción como por la forma en la que vivían sus antepasados. Las nuevas empresas también les provocan curiosidad puesto que los visitantes dan mucha importancia a poder conocer los productos, el proceso de producción, las aplicaciones así como la propia historia (Otgaar et al., 2010),

En definitiva, se puede hablar de un turismo con un gran interés tanto para la formación profesional como cultural. Todo ello podemos ofrecérselo tanto en empresas vivas como en el patrimonio que está en desuso y que hemos heredado como por ejemplo, los edificios y la maquinaria.

\section{LA PROMOCIÓN Y LA COMERCIALIZACIÓN}

El concepto de la comercialización ha comenzado a tratarse en el ámbito turístico hace pocos años atrás, a pesar de la importancia que aporta al producto y al propio destino.

Según Lobo (1998) posiblemente la actividad de la promoción ha restado protagonismo a la comercialización, creándose en la mayoría de las ocasiones un producto que nunca se ha llegado a comercializar.

De este modo ha quedado imposibilitada la posibilidad de que el cliente final pueda llegar a consumir el paquete completo, y en definitiva, el producto. Estamos por tanto ante una paralización del proceso de comercialización, siendo éste el último paso en la cadena de puesta en valor del patrimonio industrial (Pey i Cazorla, 2011).

Con los planes de dinamización y planes de excelencia se llegaron a desarrollar importantes labores de rehabilitación y puesta en valor de antiguas fábricas. Gracias a dichos planes muchos fueron los centros de interpretación enfocados hacia la explicación de la producción realizada en dichas fábricas e incluso la recuperación de maquinaria. 
Sin embargo, fue necesario que llegaran los planes de dinamización del producto turístico para que dichos recursos se convirtieran en productos y se llegara a tener presente la necesidad de comercializar. La creación de producto sí que ha sido un logro por parte de estos últimos planes pero en muchos casos se ha paralizado el proceso antes de la comercialización.

Actualmente, a pesar de dicha evolución práctica en la teoría sigue existiendo un claro problema terminológico sobre el verdadero significado del concepto comercialización en el ámbito turístico.

Por un lado, Marchena (1996) considera la promoción como aquella acción que mantiene el producto vivo y, por otro lado, la comercialización como aquella que canaliza las motivaciones de la demanda hacia una relación mercantil.

En definitiva, la promoción y la comercialización son dos conceptos diferentes aunque tenemos que tener también en cuenta que ambas son acciones que debemos de desarrollar en conjunto. La promoción por su lado ayuda a que nuestro producto sea demandado y la comercialización a ponerlo en contacto los productos o servicios con los mercados (Lobo, 1998).

En el caso de un producto de turismo industrial, y teniendo en cuenta que nos encontramos ante un producto poco conocido para la mayoría del público, es necesario que nuestro primer paso sea conseguir que el público demande nuestro producto, es decir, promocionarlo.

A continuación se resumen varios ejemplos de la promoción realizada hasta el momento de diferentes productos de turismo industrial:

1. Publicar una guía de turismo. La mayoría de los destinos optan como primer paso para dar a conocer sus productos la redacción de una guía de turismo industrial.

Por ejemplo, la Cámara Oficial de Comercio e Industria de Toledo cuenta con una Guía de Turismo Industrial donde predominan los productos artesanales y los productos agroalimentarios.

El plan de dinamización del producto turístico Ferrol-Ortegal, comentado anteriormente, a su vez ha desarrollado la misma actuación.

2. Ferias y jornadas promocionales, workshops... Han sido también una de las formas más populares de dar a conocer los productos que cada destino ofrecía. Sin embargo, cada vez más la tecnología y la web 2.0 han ido cambiando sustancialmente esta forma de promoción. Hoy en día ya podemos acceder a ferias online y por tanto es necesario también adaptar nuestra promoción a estas nuevas tendencias.

3. Fam trips. Tradicionalmente las agencias de viajes tenían mucha presencia en la comercialización y los agentes encargados de la gestión de los destinos lo sabían. Por este motivo durante muchos años se han organizado cada cierto tiempo fam trips para agentes de viajes.

Hoy en día, sin dejar de existir los fam trips para agentes de viajes, se llevan a cabo novedosas iniciativas como los press trips (viajes para periodistas) o 
incluso unos viajes especiales para blogueros, considerados como personas que influyen en las decisiones de los consumidores que leen su blog, tanto a la hora de escoger un determinado destino o un producto.

4. Publicidad en revistas especializadas, periódicos, páginas web... Una de las formas más utilizadas en la actualidad puesto que permite enfocar mucho más la promoción, ya bien sea en medios tradicionales o digitales.

5. Oficinas de turismo. Debido a los pocos recursos, tanto económicos como de personal, que puede llegar a tener una oficina municipal o comarcal, la promoción se basa en simple publicidad al turista ya en destino. De todos modos, y gracias a los diferentes planes de destino que se han ido desarrollando durante estos últimos años, se ha hecho promoción en el exterior aglomerando a su vez muchos más ayuntamientos y creando destinos más grandes.

6. Empresarios. Es necesario también que las empresas del lugar (ya bien estén relacionados totalmente con el sector turístico o no) contribuyan a la promoción de estos lugares.

Un buen ejemplo de cooperación es entre la firma valenciana Lladró puesto que ha firmado un acuerdo con el organismo que gestiona el bus turístico para que los jueves incluya una parada en la Ciudad de la Porcelana. De este modo son muchos más los turistas que realizarán la visita a la fábrica de Lladró, el bus turístico ofrece un producto más, el destino se reconocerá también por dicho producto y los turistas tendrán una motivación más de alargar la estancia o diversificar su demanda. Por tanto, es necesario cooperar con la cámara de comercio, agencias, asociaciones, empresas...

Además, a la hora de comercializar museos también va a ser necesario tener el apoyo de la administración puesto que la gran mayoría de dichos museos o centros de interpretación son propiedad de un organismo público, ya sea local, provincial o nacional.

7. Boca-Oreja: Considerada como la más antigua y más efectiva técnica de promoción, debemos de intentar que los consumidores hablen positivamente de nuestro producto.

8. En definitiva, es importante desarrollar una buena promoción del producto, dando también importancia a los diferentes eventos que se vayan desarrollando en relación al mismo para que siempre esté vivo.

El siguiente paso del proceso es la comercialización y por ello necesitamos la creación de un producto turístico. El producto constituye el objeto de la comercialización, girando en torno a él todos los demás elementos. Por ello, aunque tengamos un recurso turístico es necesario su conversión como producto turístico para poder comercializar.

En todo momento se deben ofrecer aquellas visitas que se pueden realizar tanto a empresas como a antiguas fábricas. Esto nos lleva a plantearnos la elección de la vía de comercialización que más se acomode a nuestro producto y a nuestro cliente. 
Hoy en día existen muchas formas de comercializar los productos y hay que estar innovando continuamente, buscando nuevas vías para llegar de una manera más fácil al cliente.

Por un lado, debemos tener en cuenta la vía que queremos utilizar dependiendo del tipo de turista al que queremos atraer o recibir: cliente individual o cliente de grupo.

Por otro lado, la comercialización tradicionalmente fue desarrollada por las agencias de viaje offline. Dichas agencias se han dedicado mayoritariamente al turismo de sol y playa aunque, cada vez más, han ido diversificando su oferta ofreciendo productos de turismo rural, turismo de naturaleza, turismo de aventura... Sin embargo, aún es necesario un mayor esfuerzo para que se impliquen en la venta de productos relacionados con el turismo industrial.

Actualmente la comercialización es mayoritariamente vía online. Por este motivo, es necesario tener un ente de gestión, público, privado o mixto, para poder comercializar el producto turístico. La mayoría de los agentes se decantan por algún tipo de sistema de gestión de reservas y de comercio online.

La comercialización también se puede desarrollar por medio de las propias oficinas de turismo, aunque son muchas las que aún se ven con problemas administrativos para poder llevar a cabo esta acción. Al igual que ocurre con la promoción, mayoritariamente la comercialización no se llega a desarrollar fuera de la comarca o región, siendo ya muy complicado realizarla a nivel nacional. Sin embargo, es necesario ampliar las fronteras en cuanto a la promoción puesto que ello influirá positivamente en la venta del producto.

Dentro de estas iniciativas la mayoría de las ciudades han optado por las tarjetas de visitante que se suelen ofrecer desde las oficinas de turismo. Por este motivo todo producto de turismo industrial debe de ofrecer en dichas tarjetas unos descuentos especiales en aquellos establecimientos que colaboren, entre ellos museos o centros de interpretación donde se desarrolle el turismo industrial. El público objetivo de esta tarjeta suele ser cualquier persona que se aloje en un hotel independientemente del tipo de reserva o incluso que consuman servicios de restauración. Los alojamientos por su parte, pueden ofrecer precios especiales por noche todo el año y paquetes de fin de semana en el que se alojen dos noches consecutivas con una visita al museo o centro de interpretación.

A continuación se exponen diferentes destinos que han intentado comercializar o comercializan sus productos de turismo industrial.

Por un lado, un buen ejemplo de comercialización del producto industrial es Xatic, una asociación que está promoviendo el turismo industrial en Catalunya. Gracias al Plan de Dinamización del Producto Turístico Industrial y de la Innovación Tecnológica de Catalunya han sido capaces de crear diferentes productos industriales y comercializarlos. Desde su propia página web www.byxatic.com o directamente llamando por teléfono permiten acceder a una central de reservas donde se pueden ver todos los productos que ofrecen. Tal y como señalan en su web, Xatic lleva desde el año 2010 planteándose como 
principal objetivo la comercialización de producto de turismo industrial y ya se han convertido en la primera central de reservas especializada en turismo industrial de España.

Por otro lado, se ha intentado crear la Red Española de Turismo Industrial (RETI). Una de las principales intenciones de dicha red era potenciar el turismo industrial como producto turístico, promocionar la oferta de turismo industrial y contribuir a su comercialización velando por la calidad del turismo industrial, entre muchos otros objetivos (Pey i Cazorla, 2011).

Fuera de España también tenemos importantes iniciativas como por ejemplo en Francia, quién potencia mayoritariamente el turismo industrial hacia pequeñas y medianas empresas de carácter artesanal aunque no faltan grandes empresas como por ejemplo, Airbus-France en Toulouse.

Además, son llamativas otras iniciativas como por ejemplo la reconversión de la antigua fábrica de regalices Zan en un museo sobre el mundo de los caramelos Haribo, en la región de Languedoc-Roussillón.

Francia es también conocida por la industria de la perfumería, siendo cuna de algunas de las perfumerías más famosas del mundo, como por ejemplo La Maison Guerlain. Uno de los museos más visitados en relación a esta temática es el museo del perfume en Grasse, donde se propone descubrir la historia de los perfumes, las etapas de su creación y sus frascos, cursos sobre los materiales, técnicas y química... así como varias exposiciones interactivas. Al turista se le da la oportunidad de visitar en un mismo recorrido las destilerías de Grasse: Fragonard, Galimard y Molinard.

Finalmente, destacar la Ruta Europea del Patrimonio Industrial que une a los países de Alemania, Luxemburgo, Bélgica y Reino Unido. Su principal objetivo es "proteger los sitios del patrimonio industrial europeo y usar su preservación como un motor de desarrollo para las regiones que a menudo sufren de un declive económico" (ERIH, 2007).

\section{CONCLUSIÓN}

La comercialización del producto turístico apenas se ha llegado a desarrollar hasta hace poco más de diez años. Si bien es cierto que hubo una gran evolución y cada vez más se comercializan de manera eficaz los productos turísticos. Pero para ello, como se ha ido comentando en el presente artículo, es necesario seguir una serie de pasos para realizar una comercialización eficaz de nuestro producto.

Es importante señalar también en este punto la importancia de una constante colaboración y cooperación entre los agentes encargados del patrimonio industrial y los del sector público para que los paquetes turísticos enfocados al turismo industrial tengan éxito en el mercado. 


\section{BIBLIOGRAFÍA}

Castillo Canalejo, A.M.; López-Guzmán Guzmán, T.J.; Millán Vázquez de la Torre, G. (2011): "Delimitación conceptual y consideraciones en torno al turismo industrial minero". Turydes, vol. 4, n' 9 .

European Route of Industrial Heritage, ERIH (2001): European Route of Industrial Heritage.

Fernández Zambón, G. y Guzmán Ramos, A. (2005): Patrimonio industrial y rutas turísticas culturales: algunas propuestas para Argentina. Universidad de Murcia. Cuadernos de Turismo, no 15 pp. 97-112

García, A. y Alburquerque, F.J. (2003): "El turismo de playa y el del sol y playa: ¿Sustitutivos o complementarios?”, Cuadernos de Turismo, n 11, pp. 97-105

García García, D. A. y Rivas Badillo, N. (2007): Usos turísticos del patrimonio industrial ferroviario en la ciudad de Puebla. Tesis Licenciatura. Universidad de las Américas Puebla.

Hospers, G. (2002): Industrial Heritage Tourism and Regional Restructuring in the European Union. European Planning Studies, 10 (3), pp. 397-404

Lobo Montero, P. (1998): La comercialización turística de las ciudades históricas españolas. Ería. Revista Cuatrimestral de Geografía, n 47, pp. 281-298

Marchena Gómez, M. (1996): "La promoción y comercialización de los productos turísticos desde el desarrollo regional”, en Valdés Peláez, L. y Ruiz Vega, A.V. (coord.) Turismo y promoción de destinos turísticos: implicaciones empresariales, Universidad de Oviedo, Servicio de Publicaciones, pp. 241-255.

Nácher, J. (1997) : “Turismos alternativos, cooperación y desarrollo rural: Un análisis crítico" C.I.R.I.E.C. España. Revista de Debate sobre Economía Pública, Social y Cooperativa. N N $^{\circ}$ 27. pp. 69-86

Otgaar, A.; Van den Berg, L.; Berger C.; Xiang R. (2010): Industrial tourism: Opportunities for City and Enterprise. Ashgate.

Plan de desarrollo turístico del Eume, (1998-2013): Asociación Euroeume.

Plan de dinamización del Producto turístico Ferrol-Ortegal (2006). Diputación de A Coruña.

Pardo Abad, C. J. (2004): "La reutilización del patrimonio industrial como recurso turístico. Aproximación geográfica al turismo industrial”. Treballs de la Societat Catalana de Geografía, nº 57, pp. 7-32.

Pey i Cazorla J.M. (2011): Turismo y Patrimonio Industrial. Un recurso en tiempos de crisis. Curso de verano UAM.

Valls, J.F.; Bustamante, X.; Guzmán, F.; Vila, M. (2004): Gestión de destinos turísticos sostenibles. Barcelona: Gestión 2000.

Páginas web:

http://es.franceguide.com

www.byxatic.com 
www.france.fr/es/conocer/economia/panorama/evenement/semana-de-la-industriaobjetivo-revalorizar-la-imagen-de-la-industria-francesa

www.icte.es

www.incuna.es

www.iso.org

www.lladró.com

www.patersonhistory.com/industry/inside.html 
